\title{
Development of the Control System in Bearing Roundness Measuring Instrument Based on CPAC Yang $\mathrm{Liu}^{1, \mathrm{a}}$, Ziyue $\mathrm{Wu}^{2, \mathrm{~b}^{*}}$, Tancheng $\mathrm{Xie}^{3, \mathrm{c}}$, and Yanwei $\mathrm{Xu}^{4, \mathrm{~d}}$ \\ ${ }_{1,2,3,4}$ School of Mechanical and Electronic Engineering, Henan University of Science and Technology, Luoyang 471003, China \\ aliuyang19911123@126.com, b wuziyuetutu@126.com, ${ }^{c}$ xietc@sina.cn \\ *The corresponding author
}

Keywords: CPAC; Otostudio; IEC61131-3; Bearing roundness error; Human-computer panel

\begin{abstract}
According to the application of bearing roundness measuring instrument in the field of bearing production, and developing a control system of the bearing roundness measuring instrument based on CPAC. The system adopted that CPAC as hardware platform combined with Otostudio as software platform to modular design. Then the system took international programming IEC61131-3 as standard to complete data collection, data processing, data conversion. Finally the result was displayed in friendly human-computer panel. After the experiment, the result shows that the system can measure effectively the bearing roundness error. Moreover, compared with similar products, the system has more detection efficiency and lower cost.
\end{abstract}

\section{Introduction}

It is well known that bearing is "industrial joint", the quality of the bearing directly determines the work performance of machine. In order to ensure the quality, before they leave the factory, bearing's quality need to be tested according to the related technical standard, and roundness error detection is one of the parts.

Based on the hardware platform of CPAC and software platform of Otostudio, the developed control system has good characteristic, which includes openness, extensibility, portability, operating performance and human-computer interface. So the control system can reflect the measuring results accurately and intuitively.

\section{Mechanical Structural Design}

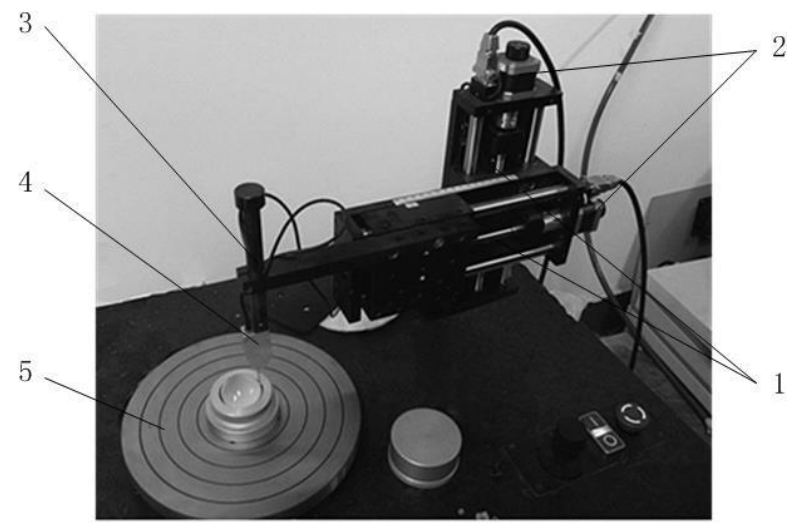

Figure 1. mechanical structural of Bearing Roundness Measuring Instrument

Mechanical structure of the bearing roundness measuring instrument is made up of five parts, as is shown in Fig.1. In the Fig.1, 1 is Lead screw. 2 is servo motor. 3 is sensor fixed frame. 4 is sensor of which type is contact displacement - OMRON_D5V model. 5 is rotary worktable. 


\section{Design of the Hardware in the System}

CPAC is the combination of the PC and motion control technology. CPAC uses CPU of the Intel X86 standard architecture and chipset as the system processor, and which adopts the high performance DSP and FPGA as coprocessor of motor control [1]. CPAC can achieve high performance multi-axis coordinated motion control and high speed control of point to point motion, at the same time, CPAC can realize all the basic functions of ordinary PC [2].

The controller chooses GUC series of GUC- 400-TPV-MOX-L2-CPAC, GUC series controller not only has high reliability, stability, anti-interference ability, and lower cost [3]. The controller has the biggest output frequency is $1 \mathrm{MHz}$, the control cycle is $200 \mathrm{mu} \mathrm{s}$, and the analog output range for $-10 v \sim+10 v$. As is shown in Fig. 2.

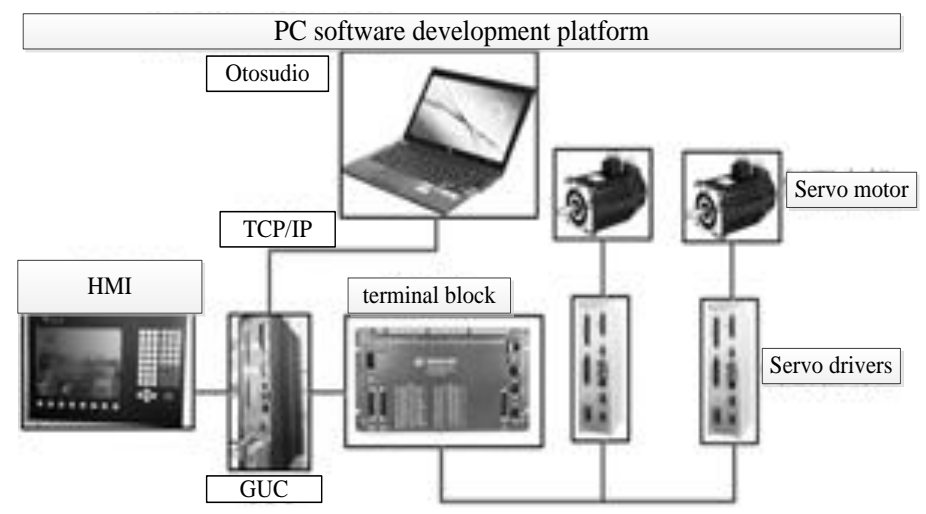

Figure 2. CPAC platform hardware structure

GUC connects to the human-computer panel and the mouse by the VGA interface and PS / 2 interface respectively. The controller connects with external terminal board through system CANOPEN bus, then terminal board connects with servo motor system and the external input output devices by cable [4].Writing a program on Otostudio software, and the Internet downloads the program to GUC directly after compiling without error. After GUC processes the program, the results will be displayed panel.

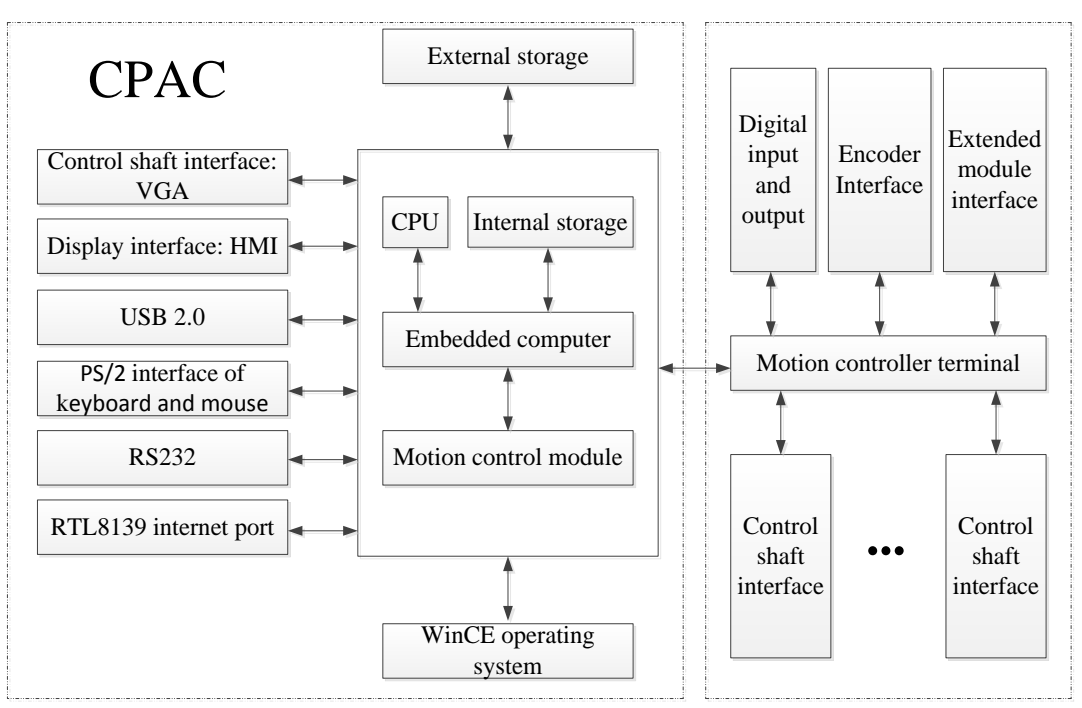

Figure 3. The principle diagram of the CPAC and terminal board 
CPAC consists of two core parts that embedded computer and motion control module. Where, the CPAC core function is realized by the motion control module that includes the motor control module, the field bus module, the I/O modules and other extensions module [4].

As shown in Fig.3, CPAC is connected to the terminal board by bus, and lead to different functions of interface on the terminal board. Such as: Control shaft interface, digital input interface, digital output interface, RS232 debugging interface debugging (not users), auxiliary encoder interface, extension module interfaces power supply interface of terminal board, interface of motion controller [4].

\section{Design of the System Software}

Software Platform. CPAC software platform is Otosudio that adopts standard of IEC61131-3 programming environment. Modular programming not only increase the readability and portability of the program, but also lower error rates, and improve efficiency, and setting up a framework of control system by the standard of IEC61131-3 [2,5].

Control System Structure. The control system of bearing roundness measuring instrument according to the function can be divided into four modules: motion control, data processing, monitoring and human-computer panel, as shown in Fig.4.

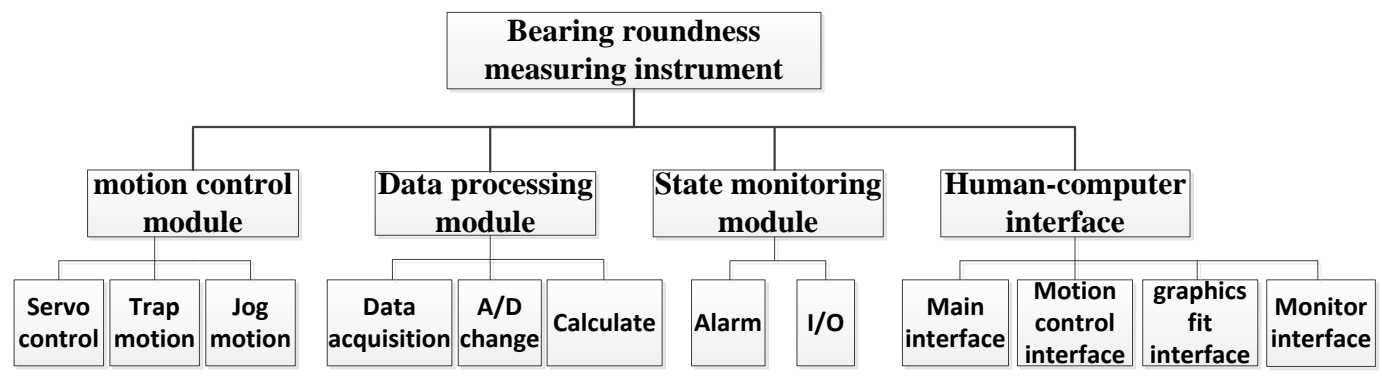

Figure 4. The control system structure of bearing roundness measuring instrument

The motion control module can be divided into three parts. The first part is the Trap motion (point-to-point motion), motor realizes displacement according to the predetermined mode by setting the parameters that number axis, velocity, target point, acceleration, deceleration, smooth time and jumping velocity of the motor in the program[6]. The second part is the Jog motion, motor realizes uniform motion in the condition of manual by set the parameters that exclusive of target point [6]. The third part is the servo control, the motor is powered, and in the preload state before the servo motor motion.

Data processing module is divided into three parts of data acquisition, A/D conversion and calculation. Data acquisition unit obtains the analog data of sensor produces by the first analog input channel [7]. A/D conversion unit is used to transform analog data to digital data. Finally, the results will be displayed in the human-computer interface through Eq.1 Eq.2 and Eq.3.

$$
\begin{aligned}
& \theta_{k}=n_{k} \pi / 5000 \\
& X_{k}=\left(R+l_{k}\right) \cdot \sin \theta_{k} \\
& Y_{k}=\left(R+l_{k}\right) \cdot \cos \theta_{k}
\end{aligned}
$$

Where, $n$ is number of pulse; $\left(X_{k}, Y_{k}\right)$ is the coordinate values of measurement point; $\mathrm{R}[\mathrm{mm}]$ is the radius of the bearing outer ring; $l$ [mm] is the radial offset; $\theta \quad[\mathrm{rad}]$ is the rotation angle; ${ }^{k}$ is the number of measurement point.

State monitoring module includes the $\mathrm{I} / \mathrm{O}$ unit and fault alarm unit. User can real-time monitoring measurement instrument by the $\mathrm{I} / \mathrm{O}$ unit and failure alarm unit will send out alarm signals to guidance the user to the cause of the problem and the location when failure occurs. 
Human-computer interface includes the main interface, the graphics fitting interface, the motion control interface and the monitoring interface. Through the human-computer interface, user can observe operation state of the measuring instrument and roundness error of the bearing.

\section{Operating Principle of the Control System}

As shown in Fig.5. There is specific workflow of the control system. Voltage value of the sensor output is changed to digital data by A/D conversion module. The digital data is transformed to displacement by the internal function of Otostudio, and calculating the rotary angle of encoder record. Finally, putting (X, Y) into the human-computer interface.

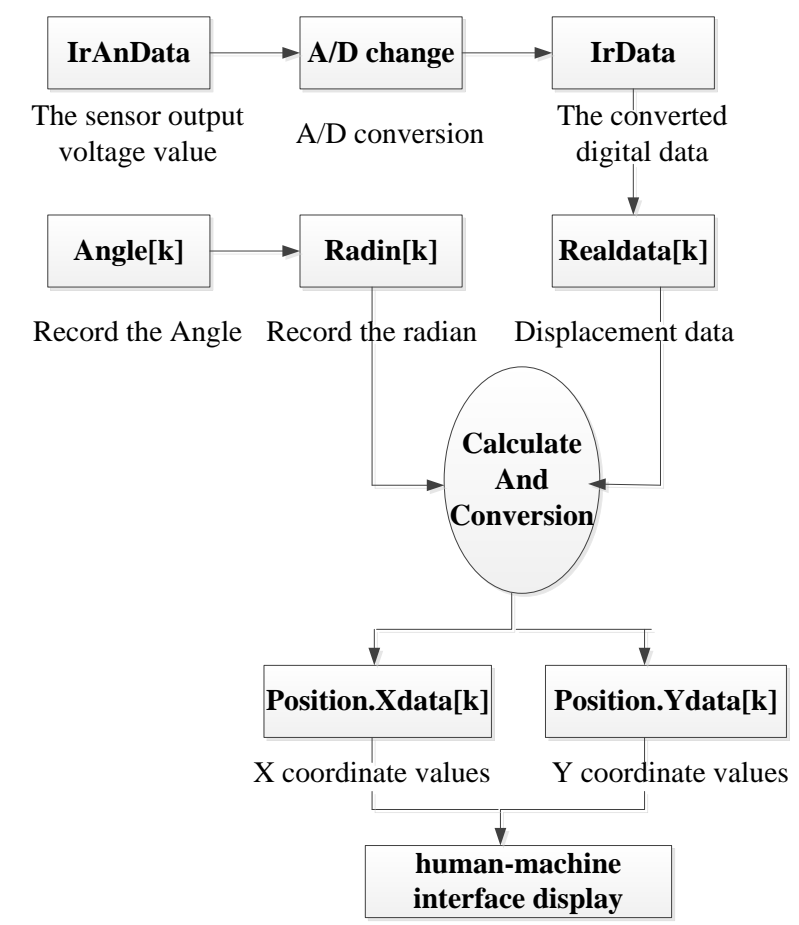

Figure 5. Operating principle of the control system

\section{Design of the Control System Program}

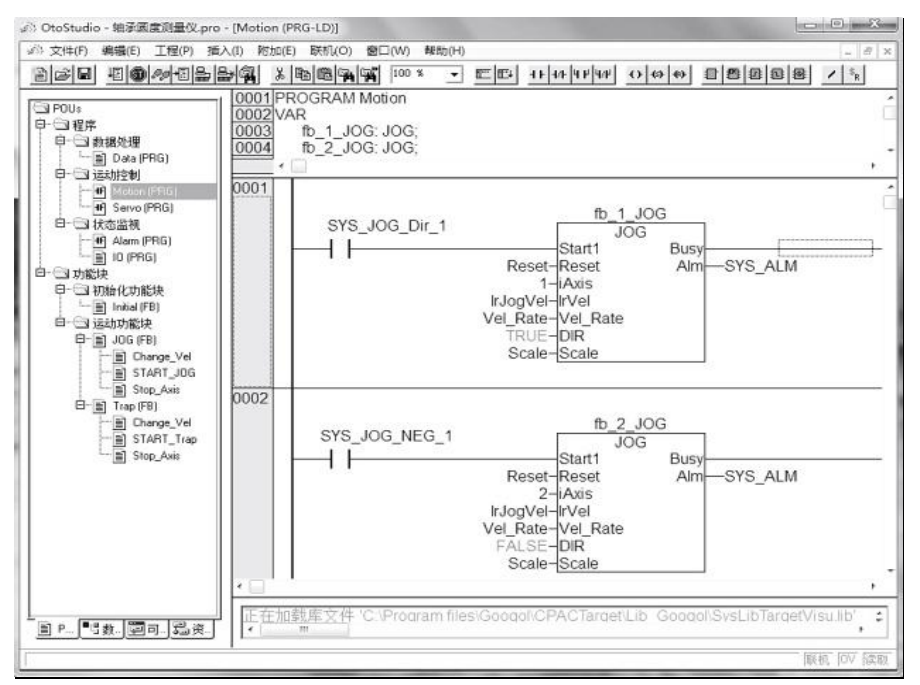

Figure 6. Program development environment of Otostudio 
Otostudio development environment as shown in Fig.6. Calling the Trap and Jog function block is used for motion control program. Inputting the bearing diameter, and probe move from origin to the point which away from the bearing outer ring $1 \mathrm{~mm}$, then calling Jog function block to adjust manually the probe contact bearings outer ring.

Importantly, data processing program is the core of the whole program. Through data processing program, the analog voltage data of probe adopts is conversed to the digital data by A/D changed module, which is combined with the data of encoder captures, and calculating the results.

There are the key codes.

rtn:=GT_GetEncPos(iAxis,ADR(EncPos), 1,0);

......

ADchange (ValEU: =IrAnologData[k], MinEU: =-10, MaxEU: =10, Raw=>IrData[k]);

RealData[k]:=IrData[k]/27648; (*the figure 27648 corresponds to $10 \mathrm{~V}$, and setting $10 \mathrm{~V}$ corresponding to $1 \mathrm{~mm} *$ )

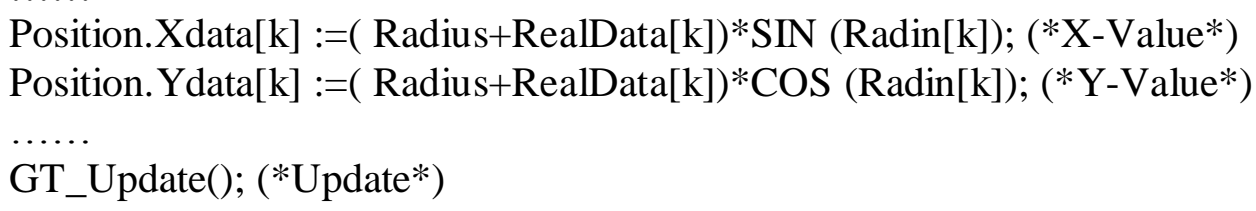

\section{The Results of System Tests}

System Operation. Control system feedbacks the motion state, fault alarm and contour fitting curve to the user through the human-machine interface, so that monitoring the measuring process of bearing roundness.

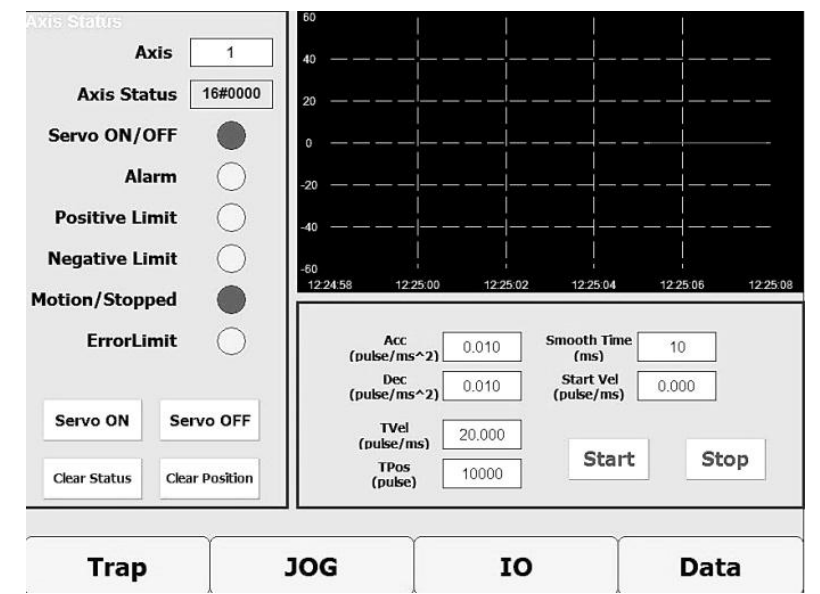

Figure 7. Main interface of Trap motion

The motion control interface is divided into the Trap (as is shown in Fig.7) and Jog, which can display motor real-time motion state, and switches freely each interface. I/O control interface contains positive and negative limit signal, alarm signal and capturing zero position signal. Graphical fitting interface is used to display the actual contour curve.

In order to ensure that the sampling data can retain truly the information of original analog data, the data sampling frequency at least twice of the original signal frequency [8], so setting sampling frequency for $40 \mathrm{~Hz}$ when programming for data acquisition. If the electric spindle speed is $20 \mathrm{n} / \mathrm{min}$ $\left(120^{\circ} / \mathrm{s}\right)$, per rotation needs $3 \mathrm{~s}$, so it can get 120 sampling points on the bearing outer ring [9].

Setting the number of sampling points in the program for 120, the angle between every two sampling points is $3^{\circ}$. Operating system after initializing, inputting bearing diameter of $40 \mathrm{~mm}$ into the graphics fitting screen, and probe will move to the point that away from bearing outer ring 1 $\mathrm{mm}$. Start the Jog motion and making the probe is contacted with outer ring of bearing. Clicking on "Start" button in human-machine interface, and clicking the "Display" button after measured. The 
final result will be displayed.

The Testing Result. Choosing the SKF production 30203J2 tapered roller bearing as test bearing, which basic parameters are as follows: $\mathrm{d}=17 \mathrm{~mm}, \mathrm{D}=40 \mathrm{~mm}, \mathrm{~T}=13.25 \mathrm{~mm}, \mathrm{~B}=12 \mathrm{~mm}, \mathrm{C}=11 \mathrm{~mm}$. As is shown in Table 1(Table 1 according to the Table 2-32 in references [10] compiles), upper deviation of the bearing outer ring is $0 \mu \mathrm{m}$, and lower deviation are $-14 \mu \mathrm{m}$. Where UD instead of upper deviation, LD instead of lower deviation.

Table1 Level 0 tapered roller bearing cylindrical tolerance (segment)

\begin{tabular}{|c|c|c|c|c|c|}
\hline $\begin{array}{c}\text { Tolerance } \\
1 \mu \mathrm{m}\end{array} \underbrace{\text { Parameter }}_{\mathrm{mm}}$ & $U D$ & $L D$ & $V_{\text {Dsp }}(\max )$ & $V_{\text {Dmp }}(\max )$ & $\mathrm{K}_{\mathrm{ca}}$ \\
\hline 1 & 0 & -12 & 12 & 9 & 18 \\
\hline $18 \sim 30$ & 0 & -12 & 12 & 9 & 18 \\
\hline $30 \sim 50$ & 0 & -14 & 14 & 11 & 20 \\
\hline $50 \sim 80$ & 0 & -16 & 16 & 12 & 25 \\
\hline $80 \sim 120$ & 0 & -18 & 18 & 14 & 35 \\
\hline
\end{tabular}

Test results as shown in Fig. 8 (In order to easy observation, lines for 20 times amplification), the outermost curve is the outline that bearing of maximum limit dimension $\mathrm{D}=40 \mathrm{~mm}$, the innermost curve is the outline that bearing of minimum limit dimension $\mathrm{D}=39.986 \mathrm{~mm}$, in the middle of the curve is the actual contour curve of the bearing. In Fig.8, the instrument displays the current radial run-out at top right corner, the grey area represents circular run-out tolerance zone. As is shown in Fig.8, this bearing roundness conforms to requirements.

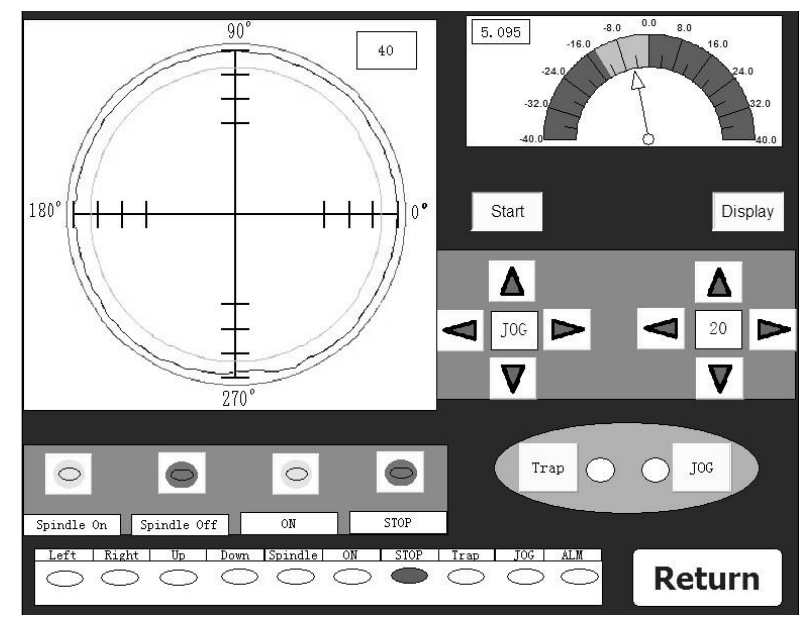

Figure 8. The graphical fitting interface

\section{Conclusion}

The control system in bearing roundness measuring instrument based on CPAC, which configuration conforms to the principle of standardization, simplification and modularization. The control system not only reduces the development cost and cycle, but also improves the detection efficiency and precision. And the control system has the better reliability, stability and anti-jamming ability. At the same, because of the openness of CPAC, improves the scalability of the system. The experiment result shows that the effect is excellent.

\section{Acknowledge}

Appreciating Natural Science Foundation of China (51305127) and scientific research key project of Henan fund (14A460018) to support this paper. In addition, thanks GoogolTech's engineer Li Xin-xing and Wang Bing to modify this paper. 


\section{References}

[1] Zhang Bao-fa, Zhao Hui, Yue You-jun. Designing of Stepper Motor Control System Based on DSP [J]. Instrument Technique and Sensor. 8 (2010) 63-64.

[2] Xu Yang, Liu Jian-qun, Liu Lei. Development of six degrees of freedom open robot control system based on CPAC [J]. Manufacturing Automation. 10 (2013) 30-31.

[3] Pang Wen-zhuo, Ku Xiang-chen, Li Ji-shun. NC system research of spring coiling machine based on movement controller [J]. Manufacturing Technology and Machine Tool. 6 (2011) $56-58$.

[4] Wang Zhu, Zhu Sheng-fang, Wang Tian-lei. Design of Automated Warehouse Control System for Bank Field Based on CPAC [J]. Machine Tool and Hydraulics. 11 (2014) 154-155.

[5] Zhou Ni-na, Wang Li, Yin Le. Design of Intelligent Traffic Lights Based on CPAC [J]. Machinery and Electronics. 6 (2016) 65-67.

[6] Wang Wen-cheng, Li Jian. Design of Motor Speed Measurement System Based on Single Chip Microcontroller [J]. Instrument Technique and Sensor. 8 (2011) 70-72.

[7] O.V. Zakharov, I.N. Bobrovskij, A.V. Kochetkov. Analysis of Methods for Estimation of Machine Workpiece Roundness. 2nd International Conference on Industrial Engineering (ICIE). Chelyabinsk, Russia. Elsevier Science BV. Amsterdam. 150 (2016) 963-968.

[8] $\mathrm{Hu}$ Wen-xiang, Du Ze-sheng. Measurement Information Signal Analysis in Mechanical Engineering [M]. The second edition. Wuhan: Huazhong University of Science and Technology Press. (2005) 81-145.

[9] H. Gafsi, G. Goch. Semi-optical in-process-metrology to determine the roundness deviation of bearing rings during heat treatment. Materialwissenschaft Und Werkstofftechnik. 43 (2012) 125-129.

[10] Qin Da-tong, Xie Li-yang. Modern mechanical handbook of design [M].Beijing: Mechanical Industry Press. (2013) 216-218. 